\title{
基于社会网络分析法的城镇基础设施健康评价 研究一一重庆万州城区电力基础设施为例
}

黄勇 ${ }^{(1) * *}$, 肖亮 ${ }^{2}$, 胡羽 ${ }^{2}$

(1) 重庆大学山地城镇建设与新技术教育部重点实验室, 重庆 400030;

(2) 重庆大学建筑城规学院, 重庆 400030

*E-mail: 463303286@qq.com

收稿日期: 2014-03-21; 接受日期: 2014-10-28

国家自然科学基金(批准号: 51108478, 51308575)和教育部博士点基金(编号: 20110191120030)资助项目

\begin{tabular}{l|l|l} 
摘要 为提高城镇电力、交通或给排水等工程型基础设施的规划设计与健康评价能 & 关键词 \\
力, 减小因其故障而可能导致的城镇全局性破坏风险, 以重庆万州电力基础设施为例, & 城乡规划 \\
采用社会网络分析(SNA)原理和方法, 分别建构了电力基础设施现状和规划网络, 提出 & 社会网络分析(SNA) \\
了由网络完备度等指标构成的网络健康评价体系, 运用网络密度等计算模型进行了网 & 城镇基础设施 \\
络拓扑结构和运行状态的健康评价. 实证研究显示, 社会网络分析方法能够对网络拓扑 & 拓扑结构 \\
结构与网络运行稳定性进行客观评价. &
\end{tabular}

\section{1 引言}

城镇基础设施健康发展是城镇生存和发展的根 本条件. 事实证明, 电力、交通或给排水等工程型基 础设施一旦出现故障, 往往会造成城镇功能的局部 或整体丧失, 进而威胁人民生命财产安全. 这类基础 设施存在网络特性, 有率一发而动全身的放大器效 应 $^{[1,2]}$. 局部故障不仅会影响设施本身的健康运行 ${ }^{[3,4]}$, 也可能产生连锁反应 ${ }^{[5]}$, 导致其所在城镇出现全局性 问题 ${ }^{[6-8]}$. 面对城镇工程型基础设施的网络健康和安 全新课题 ${ }^{[0 \sim 11]}$, 国内外进行了大量研究 ${ }^{[12 \sim 19]}$. 其中的 关键问题之一是提高城镇工程型基础设施网络的规 划设计、健康评估和空间布局水平. 为此, 以三峡库 区中心城市重庆万州的电力基础设施为例, 创新运
用社会网络分析原理和方法，建立工程型基础设施 网络的拓扑结构, 提出健康评价体系. 并对比分析现 状和规划网络, 提出改进建议. 为科学建设城镇工程 型基础设施探索新方法.

\section{2 研究靶区}

重庆万州区位于长江中上游结合部, 是三峡库 区中心城市. 受库区移民迁建和后期扶持发展影响, 逐步成为成渝城镇群的重要节点城市和综合交通枢 纽, 是西南地区快速发展的典型城市之一(图 1). 这 也决定了万州城区电力基础设施网络荷载较大、网络 运行稳定性要求高. 2012 年, 城区现有 500 千伏变电 站 1 座、220 千伏 3 座、 110 千伏 11 座, 变电总容量

引用格式: 黄勇, 肖亮, 胡羽. 基于社会网络分析法的城镇基础设施健康评价研究一以重庆万州城区电力基础设施为例. 中国科学: 技术科学, 2015, 45: 68-80

Huang Y, Xiao L, Hu Y. Evaluation of urban infrastructure project health based on social network analysis: A case of power grid planning of Wanzhou Districts, Chongqing City (in Chinese). Sci Sin Tech, 2015, 45: 68-80, doi: 10.1360/N092014-00071 


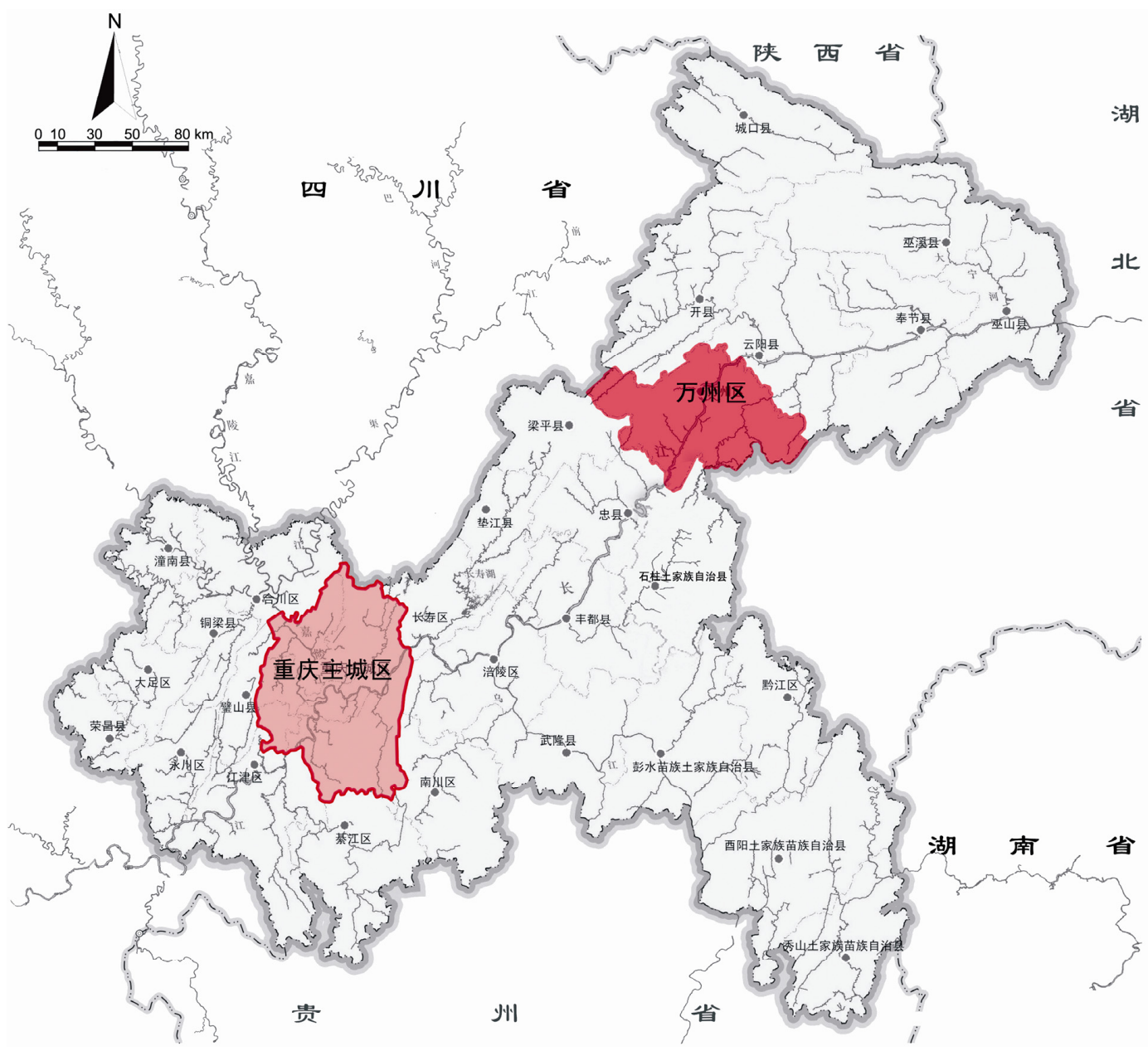

图 1 万州在重庆市的区域位置

294.05 万千伏安(图 2). 事实表明, 现状电网中主电 源荷载大, 供电能力弱; 变电站布点不足, 变电容量 偏小, 部分变电站容载比过高; 输配电网络不完善, 电网结构脆弱, 运行可靠性差. 基于上述问题, 万州 城区提出了 2020 年供电网络的初步设计方案 ${ }^{1)}$ (图 3).

\section{3 研究框架}

\section{1 技术路线}

万州城区电力基础设施网络的健康评价分三个 步骤. 第一步, 运用社会网络分析原理, 构建现状和
规划两个网络. 第二步, 根据电力基础设施健康运行 的要求和社会网络分析方法的计算模型, 建立计算 指标和评价模型. 第三步, 计算两个网络拓扑结构的 相关指标, 对比分析计算数据, 得出结论, 提出电力 基础设施规划网络的优化策略.

\section{2 研究方法及网络构建}

社会网络分析方法发源于计量社会学, 用于研 究社会活动中不同行动者的相互关系 ${ }^{[20]}$. 其基本原 理是将行动者作为“点”, 行动者之间的关系作为“线”, 从而构建一张行动者“网络”. 由于其原理清晰, 操作

1) 根据负荷预测, 在城区规划 500 千伏变电站 1 座, 220 千伏变电站 6 座, 110 千伏变电站 26 座, 加上现有及改造变电站, 至规划末 期 2020 年, 城区将形成 2 座 500 千伏变电站、11 座 220 千伏变电站及 39 座 110 千伏变电站的供电网络格局. 


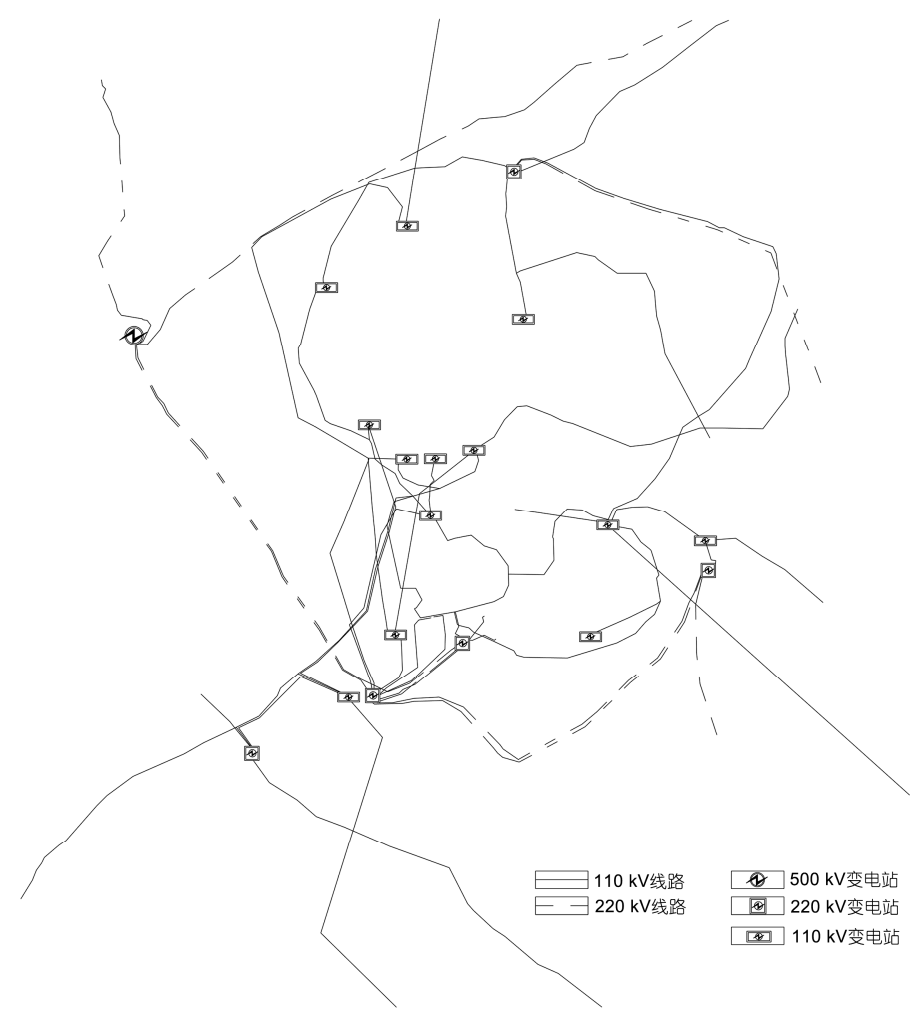

图 2 万州城区现状(2013)电力基础设施

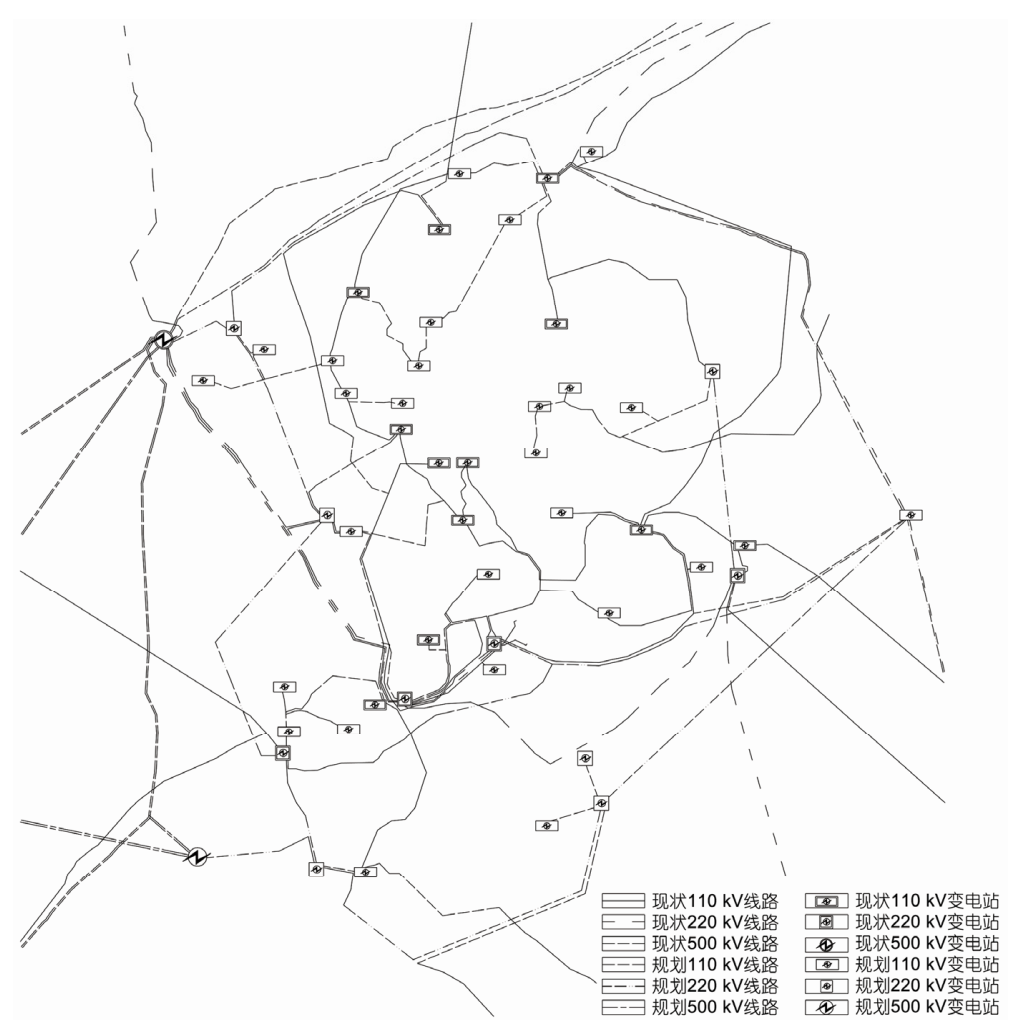

图 3 万州城区规划(2020)电力基础设施 
简单, 目前在城乡管理结构 ${ }^{[21]} 、$ 产业集群结构 ${ }^{[22,23]}$ 、 区域经济结构 ${ }^{[24]}$ 、社区社群网络 ${ }^{[25]}$ 及工业园区结构 ${ }^{[26]}$ 等领域均有运用.

在万州城区电力基础设施中, 各级变电站及其 他线路节点被认为是“点”, “点”之间的连接关系即为 “线”, “线”在一定程度上反应的是线路设施. “点”之 间存在连接关系计为“ 1 ”, 不存在连接关系计为“ 0 ”, 并通过社会网络分析软件 Ucinet 构建了现状与规划 两张空间拓扑网络关系图(图 5 和 6). 社会网络分析 模型与传统线路接线图(图 2 和 3)具有一定的相似性, 均能清晰地表达出设施网络中电器元件的位置、配线 方式和接线方式, 但社会网络分析方法能够更为直 观的表达出基础设施网络整体的拓扑关系, 涵盖网 络整体结构及单个设施的网络特性评价.

相比于国内外电力网络研究中常用的网络图模 型、Agent 模型、物理模型、系统动力学模型或投入 产出模型等传统分析方法 ${ }^{[27]}$, 社会网络分析方法擅 长于网络个体间关联关系的分析, 即能表达网络结
构的整体特征, 也能反映个体在整体的位置, 以及描 述整体对个体的影响程度. 弥补了传统分析方法缺 乏网络间关联关系分析能力的不足 ${ }^{[27]}$. 对电力等工 程型基础设施网络的健康评估、规划布局等问题, 更 有针对性. 另外, 社会网络分析方法的技术过程更简 单, 研究结论也更简明 ${ }^{[28,29]}$.

\section{3 评价模型与指标}

通常, 工程型基础设施网络的健康程度取决于 网络自身的拓扑结构合理性和运行状态的效率与稳

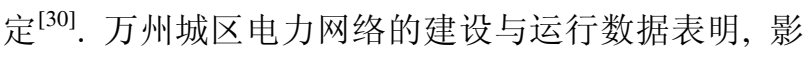
响网络结构的健康因素有网络完备度和凝聚度、网络 层级关联度、局部稳定成份比例以及网络节点脆弱性 等 4 个方面. 网络健康运行则应该维持荷载在整个网 络中的均衡分布, 避免容载比过高. 社会网络分析方 法中的密度、凝聚子群、 $K$-核、Lambda 集合、切点 和中心度等计算模型可以对这些指标予以对应的定 量计算. 由此, 建立健康评价模型和指标(图 4).

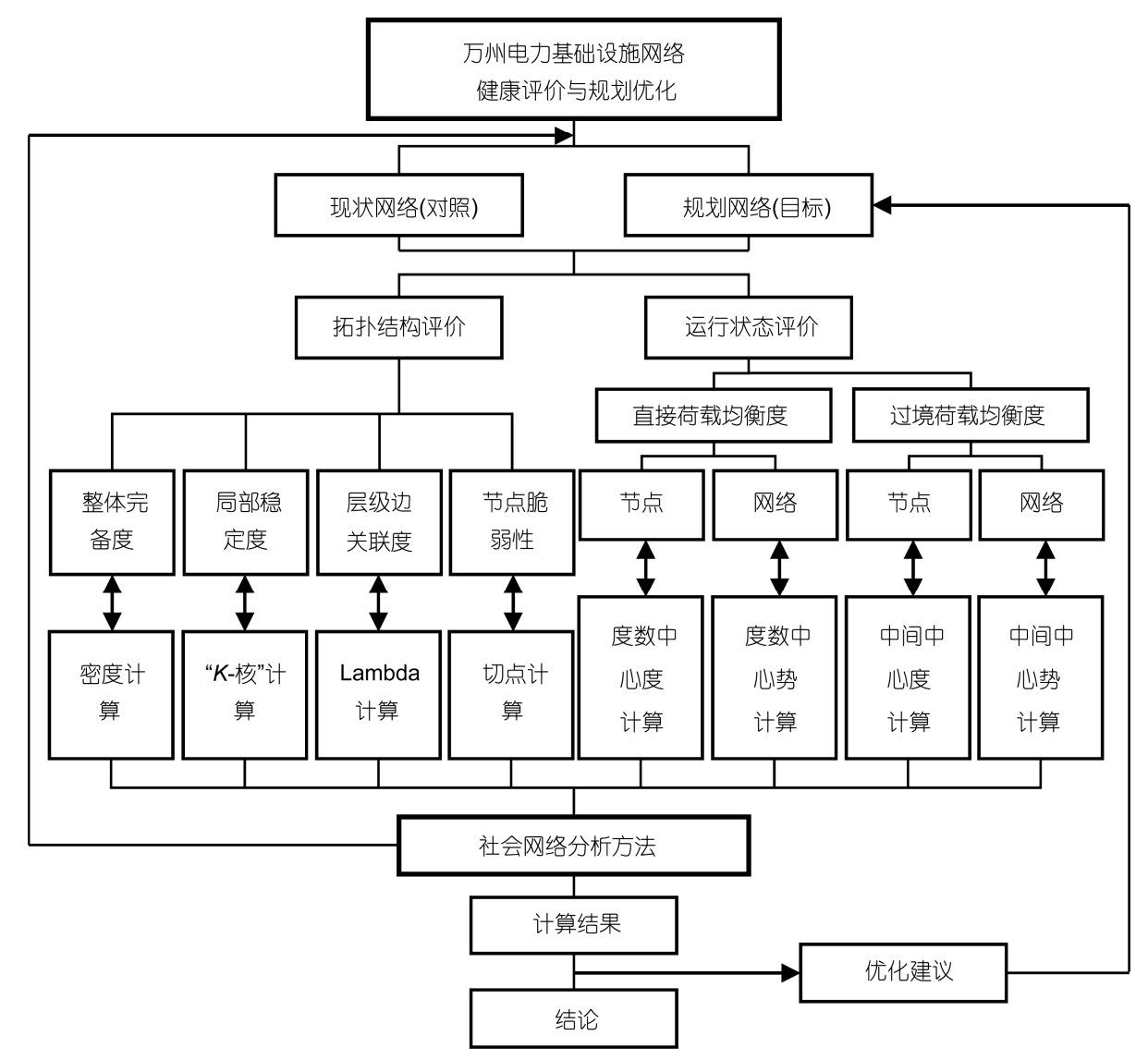

图 4 电力基础设施网络健康评价的技术模型 


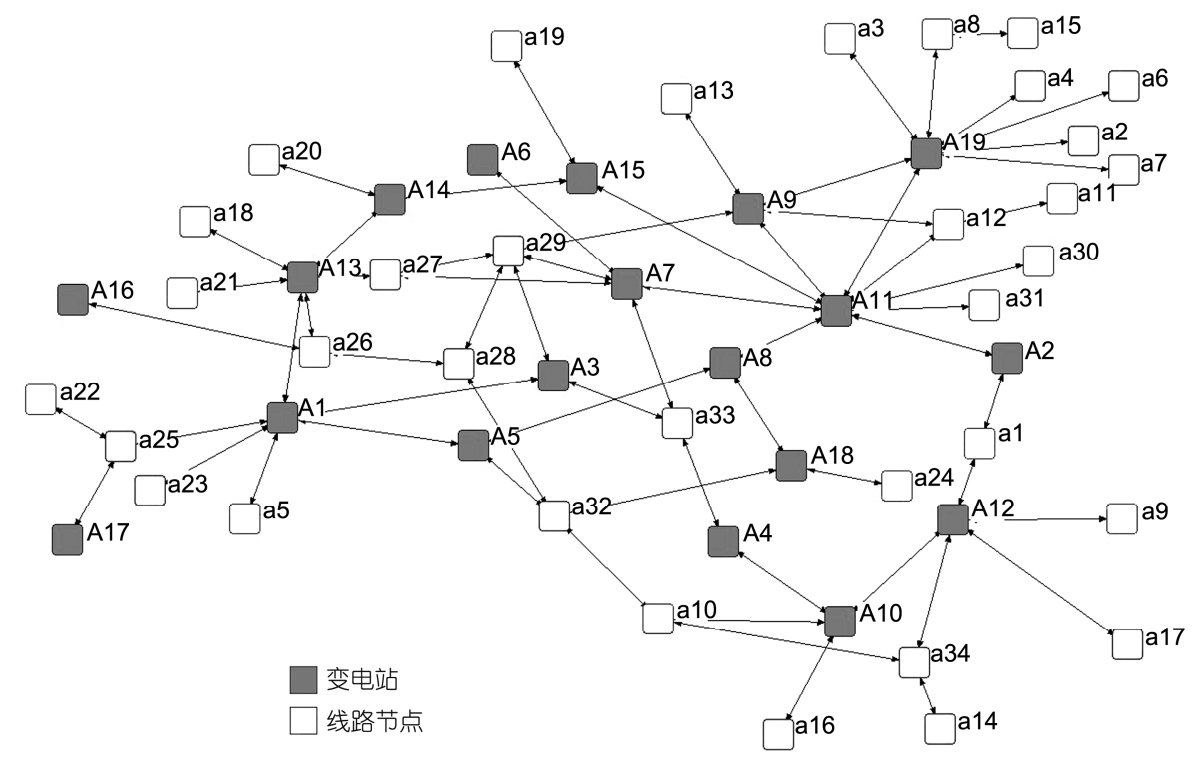

图 5 现状 $220 \mathrm{kV} / 110 \mathrm{kV}$ 电力网络

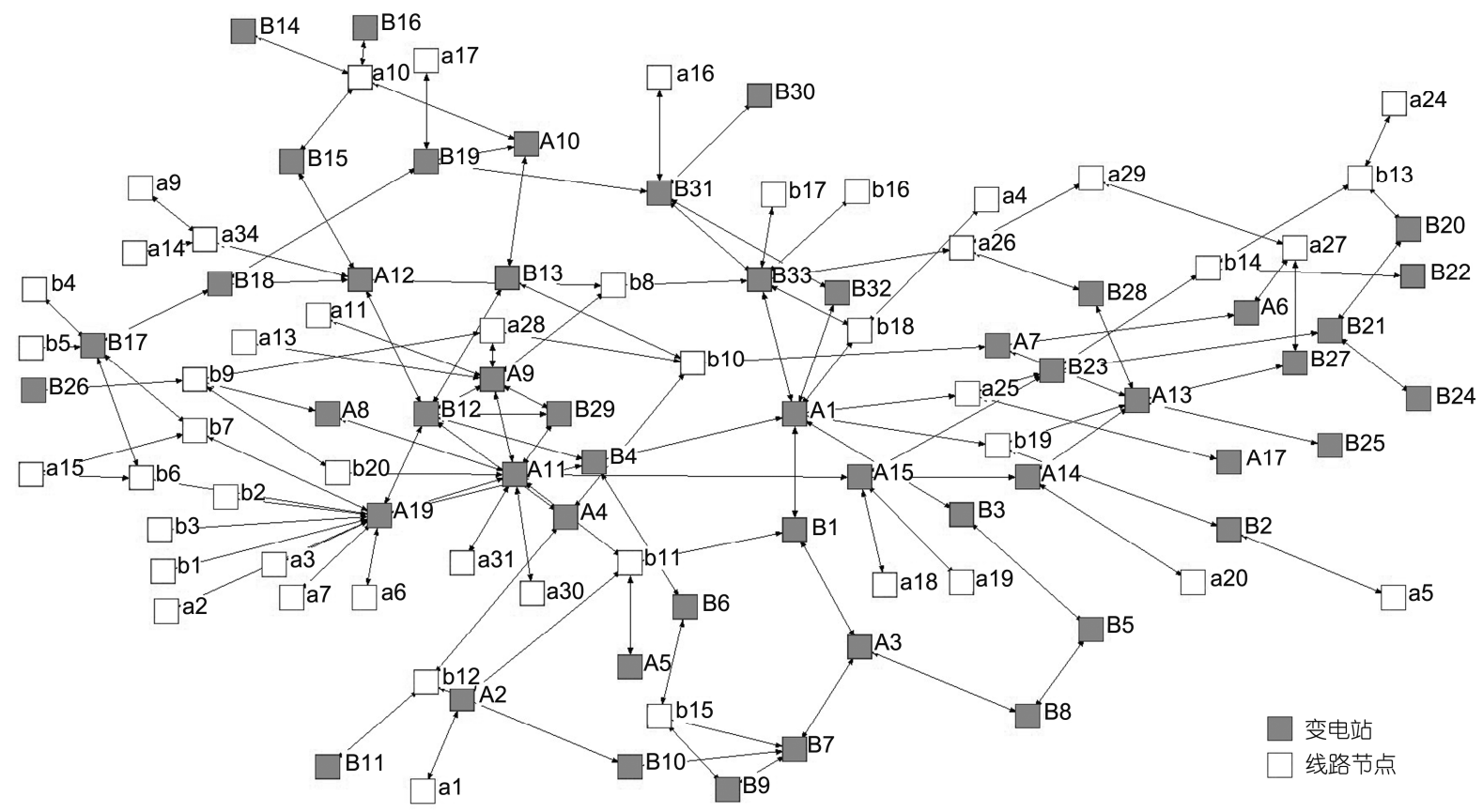

图 6 规划 $220 \mathrm{kV} / 110 \mathrm{kV}$ 电力网络

\section{4 计算过程}

\section{1 网络拓扑结构评价}

1) 网络整体完备度

网络密度公式可以测定网络整体完备程度, 计 算公式为

$$
P=L /[n(n-1) / 2],
$$

式中, $P$ 为网络密度, $L$ 为网络中实际存在的连接数, $n$ 为网络中实际存在的节点数.

现状电网整体完备度为 0.0493 , 凝聚力系数为 0.308 , 节点间平均距离为 4.038 . 规划电网整体完备 度为 0.0271 , 凝聚力系数为 0.277 , 节点间平均距离 
为 4.245. 由此, 现状电力网络的整体完备度及凝聚 力系数均小于规划, 节点间平均距离大于规划, 说明 现状电力网络的完备度优于规划(图 5 和 6).

2) 网络局部稳定度

“ $K$-核” $(K=1,2,3 \cdots)$ 是建立在点度数上的凝聚子 群概念, 表达一个子图中的全部点至少与其他子图 中的“ $K$ ”个他点相连. 由此, “ $K$ ”值越高、“ $K$-核”占比 越高, 则该网络的局部稳定成分越多, 网络整体也就
越稳定.

现状电网中，“2-核”共有 19 个，占网络整体的 $35.85 \%$, 其中变电站节点 9 个, 线路节点 10 个. 规划 电网中, “2-核”共有 58 个, 占网络整体的 $59.79 \%$, 其 中变电站节点 35 个, 线路节点 28 个. 规划电网的“2核”成分明显高于现状，局部稳定性有较大提升(图 7 和 8). 同时发现, 变电站 A17 在现状与规划电网中都 不是“2-核”成分，急需提高其稳定性.

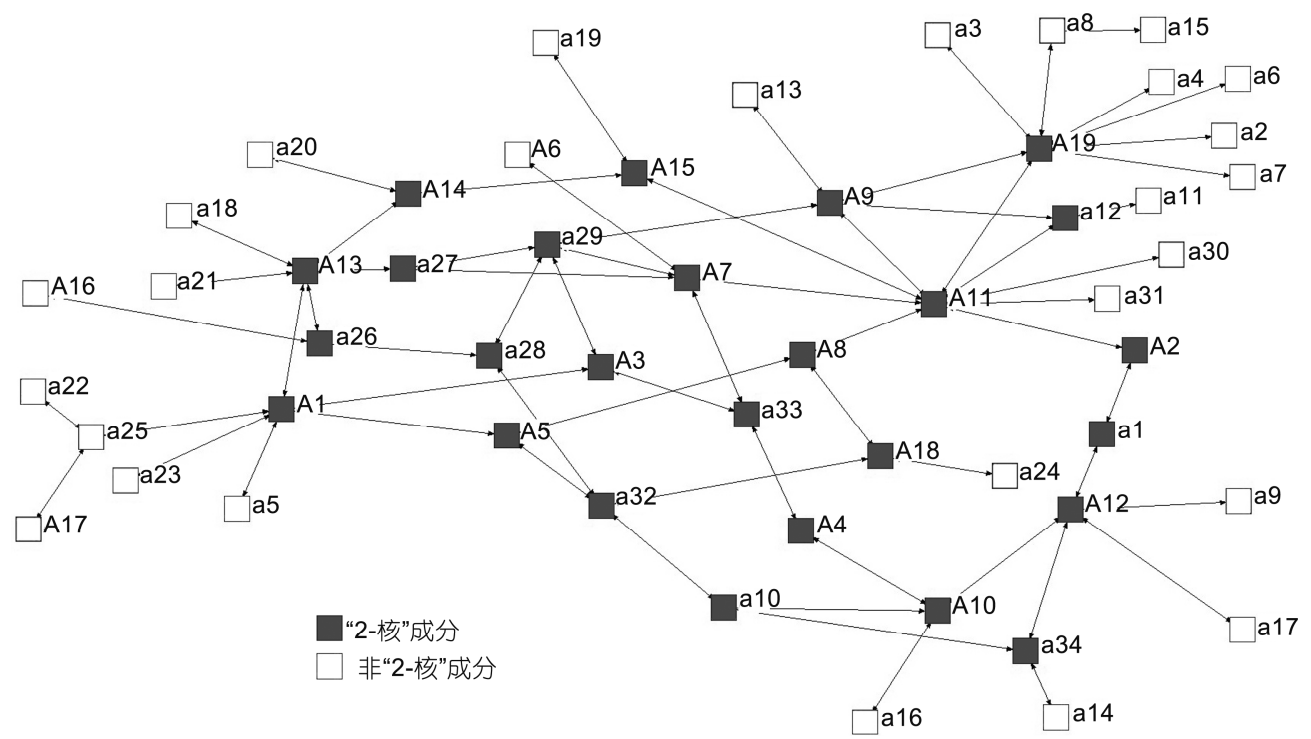

图 7 现状电网“2-核”分布

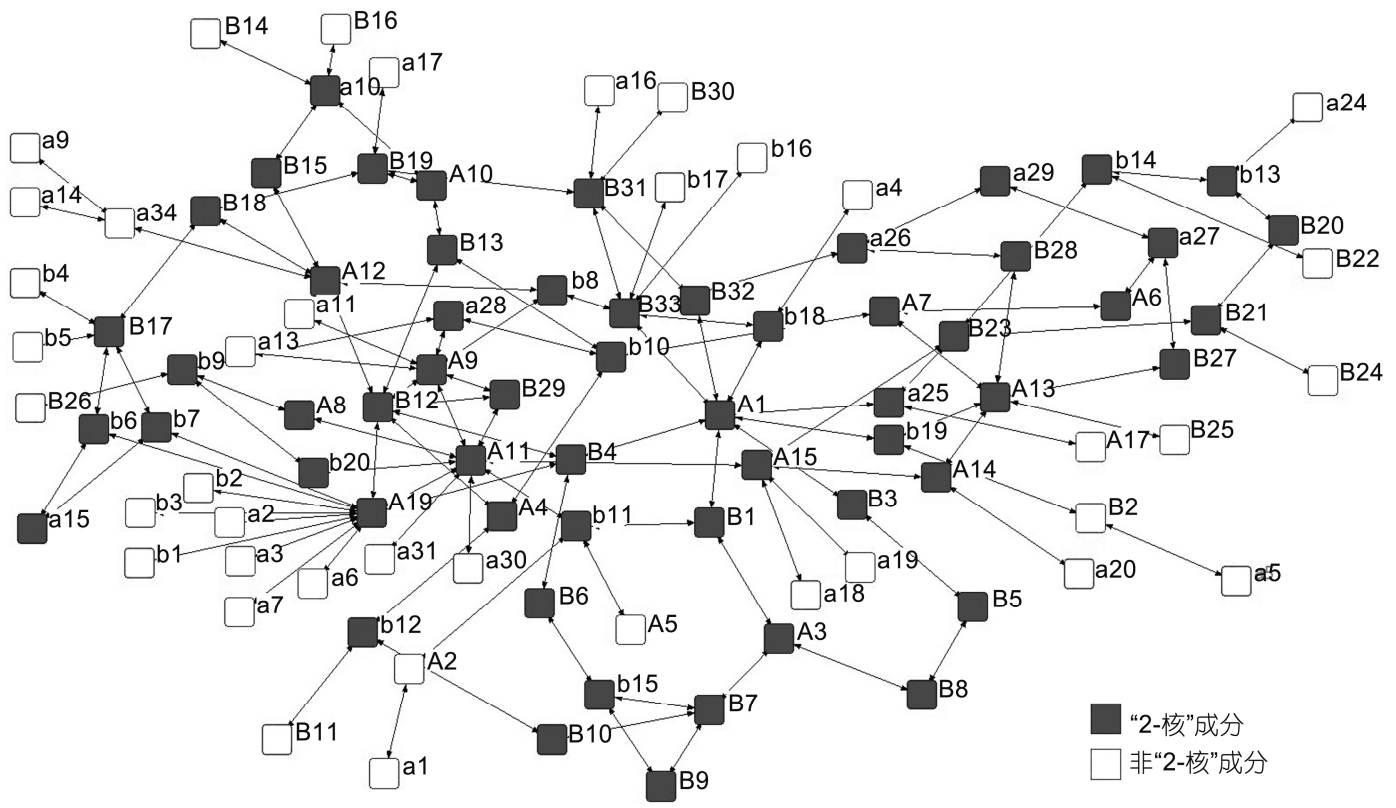

图 8 规划电网“2-核”分布 


\section{3) 网络层级边关联度}

“Lambda 集合”是网络整体结构稳定性的评价因 子, 通过网络中某 2 个点的“边关联度”指数给出定量 测量. 边关联度指数可标记为 $s(i, j)$, 等于为了使得 这 2 个点之间不存在任何路径, 必须从图中去掉的线 的最小数目, 该值越大二者之间的关系越稳健.

由 Ucinet 软件计算可知, 现状电网有 1, 2, 3, 4 和 5 等五个级别, 边关联比例分别为 $100 \%, 50.94 \%$, $33.96 \%, 11.32 \%, 3.77 \%$; 规划电网有 13 个级别, 边关 联比例分别是 $100 \%, 73.20 \%, 54.64 \%, 37.11 \%, 27.83 \%$, $20.62 \%, 11.34 \%, 8.25 \%, 6.19 \%, 5.15 \%, 4.12 \%, 3.09 \%$ 及 $2.06 \%$. 对比发现, 现状电网 $50.94 \%$ 的边关联分布 在“2”以下，规划电网 $54.64 \%$ 的边关联分布在“4”以 下. 由此, 规划电网的边关联度指数 $(s)$ 更高, 整体稳 定性优于现状(图 9 和 10).

4) 网络节点脆弱性

“切点”是网络中联系的关键节点. 一旦去掉, 网 络将分离成多个独立的部分. 分析切点在整个网络 的占比, 可衡量网络的节点脆弱程度. 计算可知, 现 状电网中“切点”占比为 $30.19 \%$, 规划电网为 $25.77 \%$. 两者在节点脆弱性上的差异性不明显(图 11 和 12).

\section{2 网络运行状态评价}

为避免网络局部出现过载, 需要使直接荷载与
过境荷载两种不同类型的电力荷载在网络中处于均 衡分布的状态, 需要对网络运行中上述两种荷载的 分布情况进行评估.

\section{1) 直接荷载均衡度}

(1)节点荷载压力

度数中心度用来衡量单个点在网络中占据的核 心性, 该值越高越处于网络的中心位置, 往往也意味 着更大的荷载压力. 度数中心度可以分为两类: 绝对 中心度与相对中心度, 前者根据与节点直接相连的 节点数目, 测量出来的中心度可称为 “局部中心度 (local centrality)”, 后者是前者的标准化, 适用于对 不同规模的网络进行横向比较, 本研究选择相对中 心度作为衡量节点荷载压力的指标. 公式为

$$
D s=s /(n-1),
$$

式中, $D s$ 为相对度数中心度, $s$ 为与某一节点直接相 连的对象数, $n$ 为节点数.

计算可知, 现状电网 A19 (500 kV), A11(220 kV) 的相对中心度最高, 其值分别为 0.19 和 0.17 ; 其次 $110 \mathrm{kV}$ 变电站节点 $\mathrm{A} 1, \mathrm{~A} 9, \mathrm{~A} 13, \mathrm{~A} 12, \mathrm{~A} 7$ 及线路节点 a29, 其值分别为 $0.11,0.09,0.09,0.09,0.09$ 和 0.09 . 上述节点相对其他节点而言, 面临着更大的荷载压力. 规划电网 $\mathrm{A} 19(500 \mathrm{kV})$ 的相对中心度最高, 其值为 0.22; 其次 $\mathrm{A} 11(220 \mathrm{kV}), \mathrm{A} 1(110 \mathrm{kV}), \mathrm{A} 9(110 \mathrm{kV}), \mathrm{B} 12$ $(220 \mathrm{kV}), \mathrm{B} 33(220 \mathrm{kV}), \mathrm{A} 13(110 \mathrm{kV})$ 和 A12 $(220 \mathrm{kV})$,

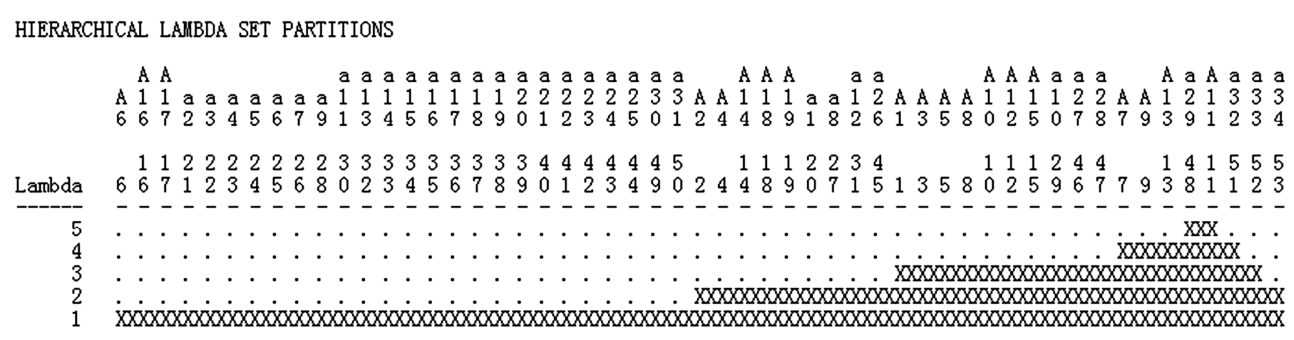

图 9 现状电网“Lambda 集合”分布图

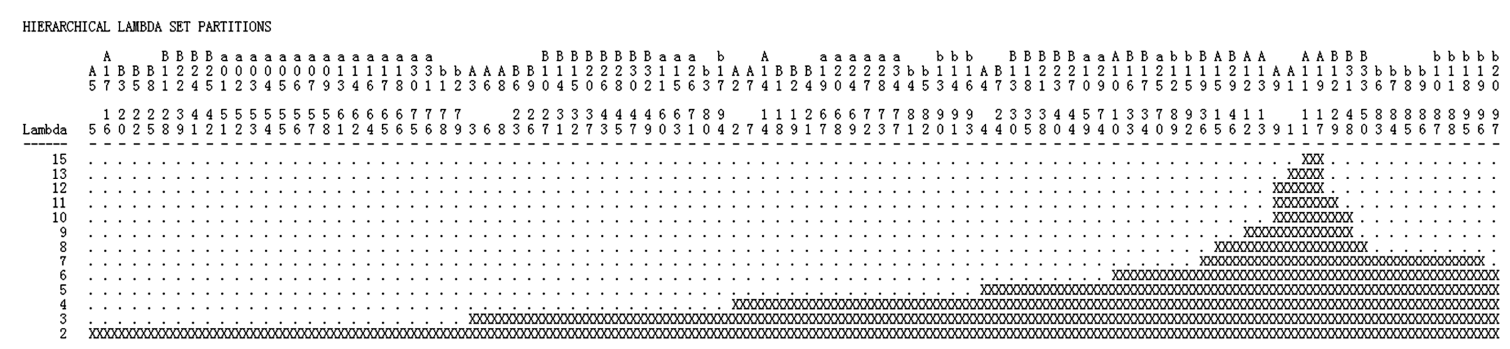

图 10 规划电网“Lambda 集合”分析图 


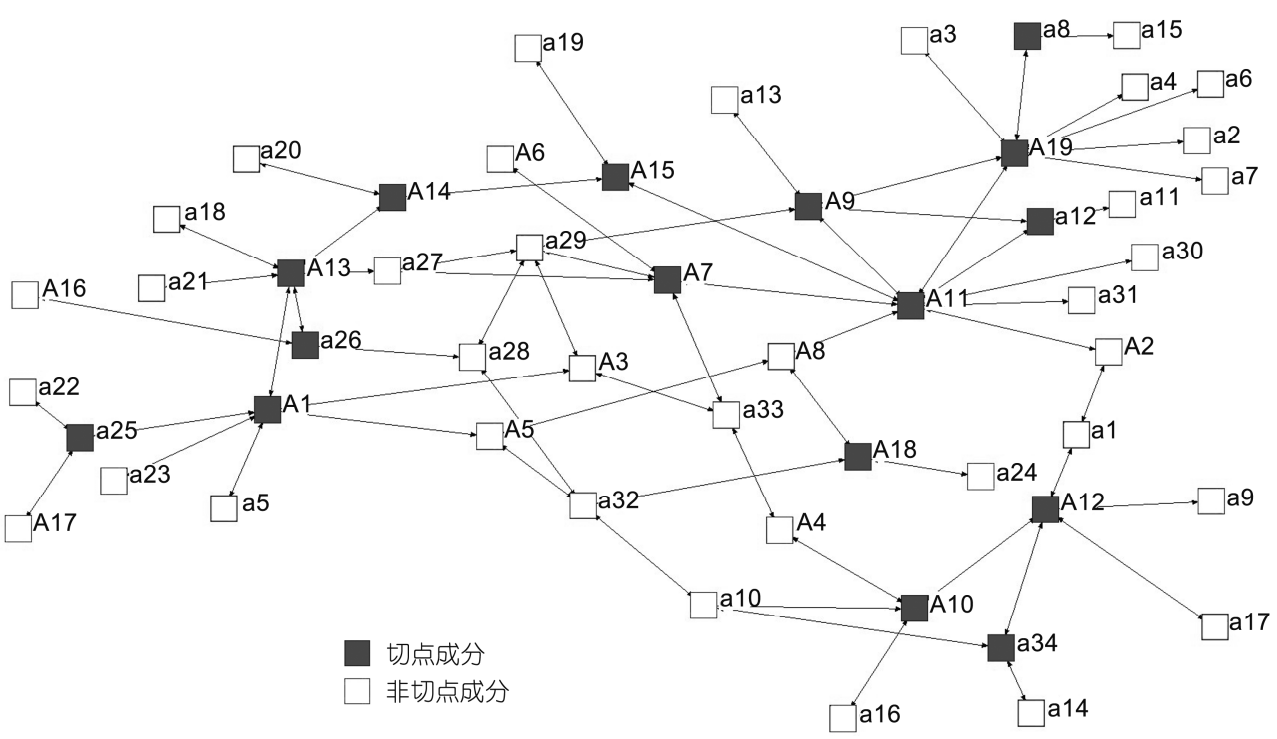

图 11 现状电网切点分布图

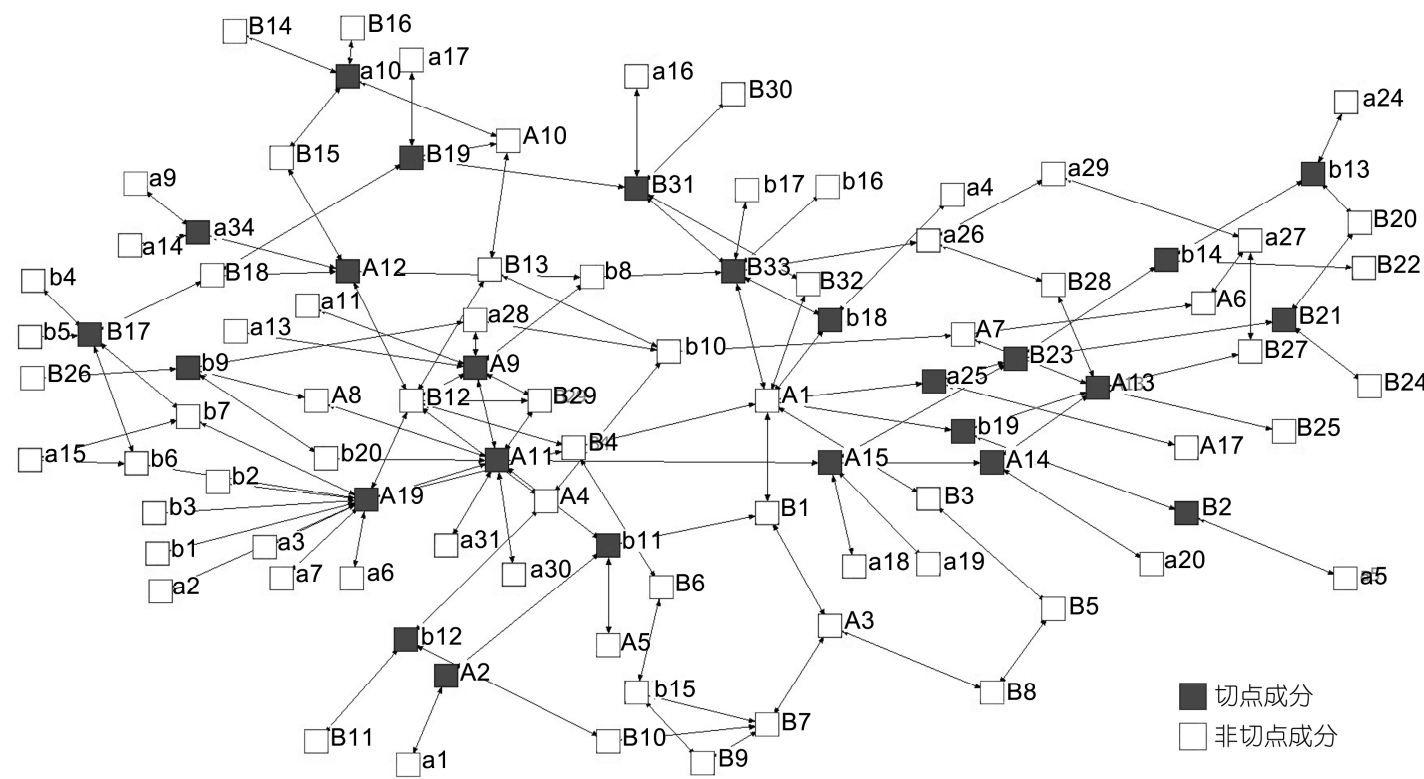

图 12 规划电网切点分布图

其值分别为 $0.15,0.13,0.12,0.11,0.10,0.09$ 和 0.09 (图 13 和 14).

分析得知, 现状电网 $\mathrm{A} 11(220 \mathrm{kV})$ 有过载风险, 虽然规划电网增加了 2 个 $220 \mathrm{kV}$ 变电站(B12, B33), 但并未降低现状 A11 的过载风险.

(2)网络荷载压力

“度数中心势”能评价网络的整体中心性,可评估 网络运行中荷载的整体分布情况, 防止网络出现局
部失衡. 公式如下：

$$
C=\frac{\sum_{i=1}^{n}\left(C_{\max }-C_{i}\right)}{\max \left[\sum_{i=1}^{n} C_{\max }-C_{i}\right]},
$$

式中, $C_{\max }$ 为网络中各节点度数中心度的最大值, $C_{i}$ 为节点 $i$ 的中心度.

计算可知, 现状电网的度数中心势为 $7.43 \%$, 规 


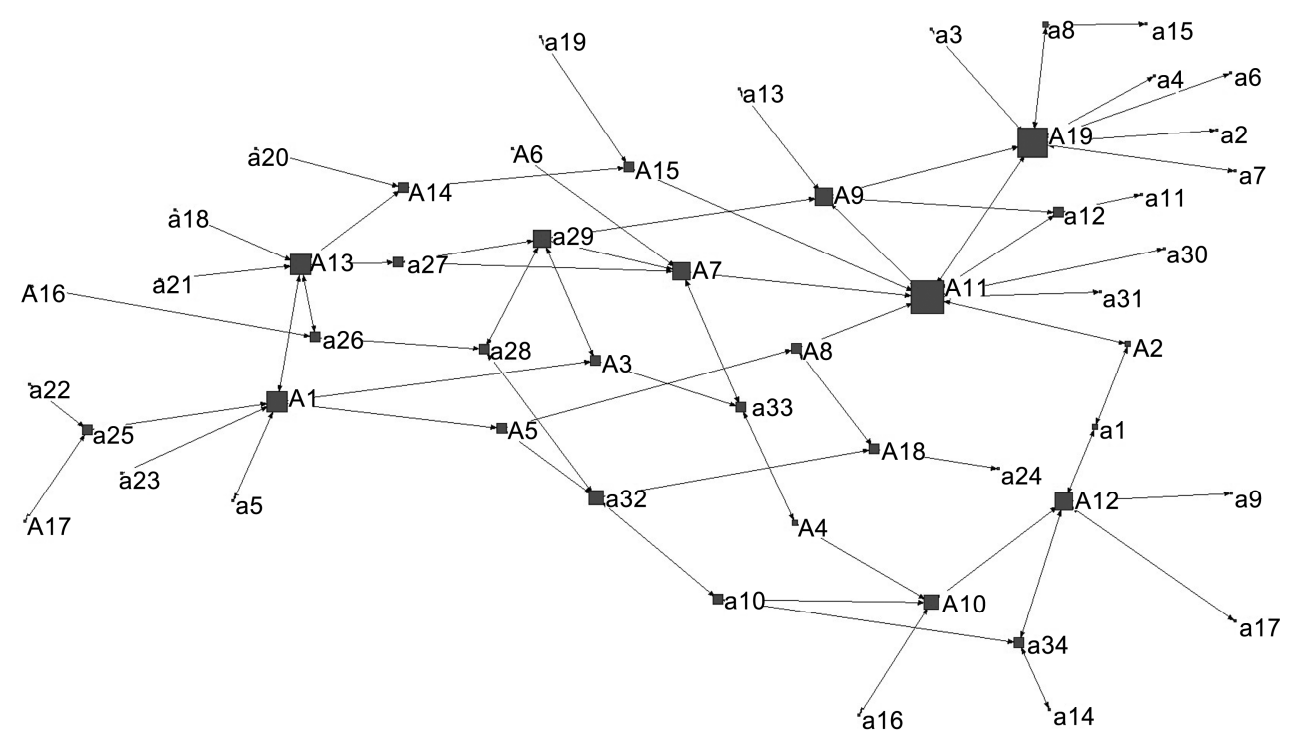

图 13 现状电网度数中心性分析

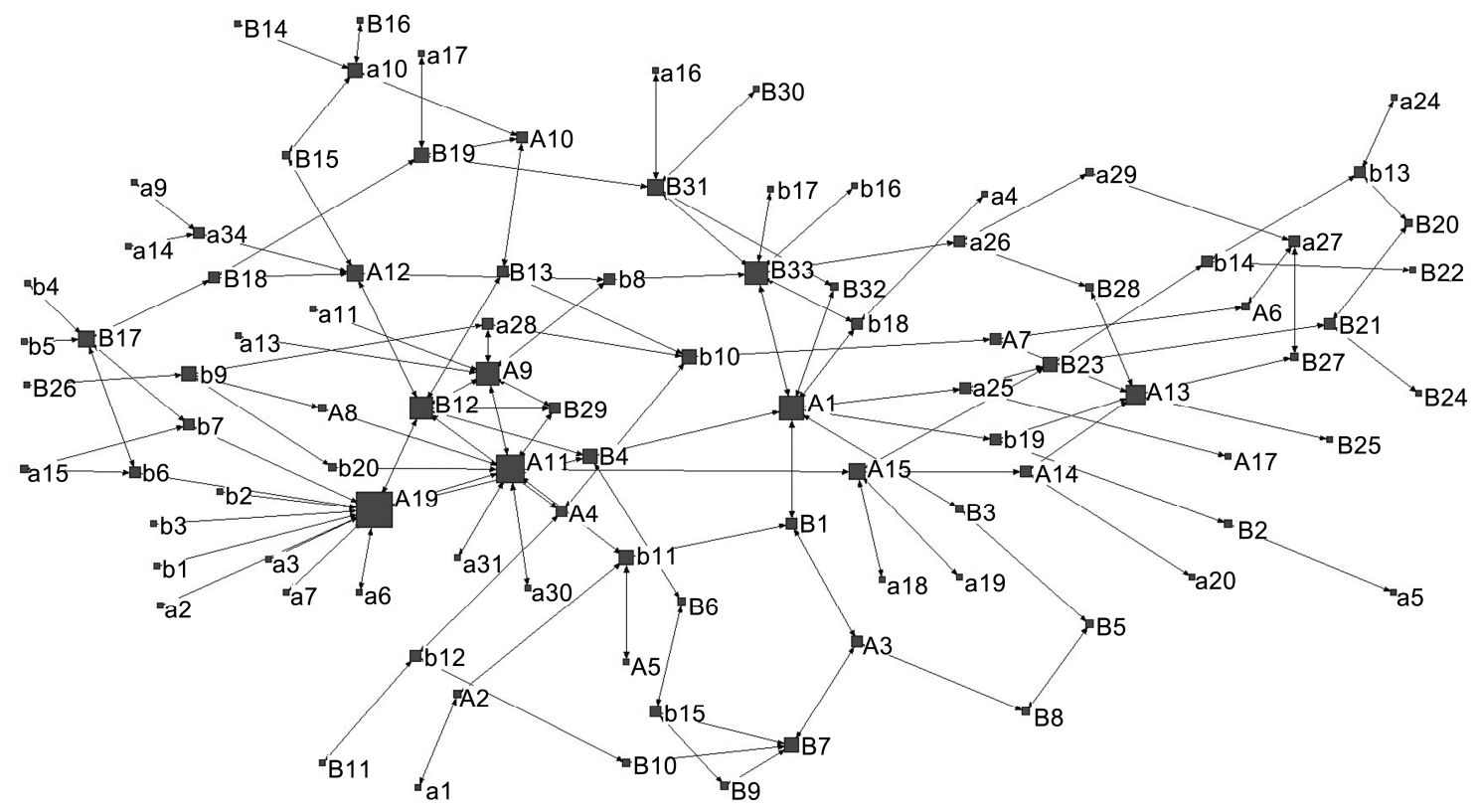

图 14 规划电网度数中心性分析

划电网为 $8.71 \%$, 两者在网络整体荷载均衡度上并无 太大的区别, 两者数值均偏小, 有较好表现.

2) 过境荷载均衡度分析

中间中心性测量的是一个点(线)在多大程度上 位于图上其他点(线)的中间. 在网络中, 中间中心性 高的点(线)需要承受更多的过境荷载压力, 容易出现 过载问题. 中间中心性主要通过“中间中心势”和“度 数相对中间中心度”予以测定. 前者适用于评价网络
整体，后者衡量单个节点的中间中心性.

(1) 网络过境荷载集中程度 “中间中心势”计算公式如下:

$$
C_{\mathrm{B}}=\frac{\sum_{i=1}^{n}\left(C_{\mathrm{RBmax}}-C_{\mathrm{RB} i}\right)}{n-1},
$$

式中, $C_{\mathrm{RB} \text { max }}$ 是点的相对中间中心度.

计算得知，现状电网中间中心势为 $36.70 \%$, 规 
划电网为 $32.91 \%$, 两者中间中心势均较大, 但规划 电网一定程度上优化了过境荷载的均衡度分布.

(2) 节点过境荷载压力

度数相对中间中心度计算公式为

$$
C_{\mathrm{RB} i}=\frac{2 C_{\mathrm{AB} i}}{n^{2}-3 n+2}=\frac{\sum_{j}^{n} \sum_{k}^{n} b_{j k}(i)}{n^{2}-3 n+2},
$$

式中, $C_{\mathrm{RB} i}$ 为相对中间中心度, $C_{\mathrm{AB} i}$ 为绝对中间中心 度, $b_{j k}(i)$ 为 $i$ 处于点 $j$ 和 $k$ 之间的捷径上的概率.

计算可知, 现状电网中, 中间中心度最高的点为 $\mathrm{A} 11(220 \mathrm{kV})$, 其值为 0.42 ; 其次 $\mathrm{A} 19(500 \mathrm{kV}), \mathrm{A} 1$ (220 kV), A13(110 kV), a29, A7(110 kV), a32, A9(220 $\mathrm{kV}), \mathrm{A} 12(220 \mathrm{kV}), \mathrm{A} 8(110 \mathrm{kV})$ 和 $\mathrm{A} 5(110 \mathrm{kV})$, 其值分 别为 $0.26,0.22,0.15,0.13,0.13,0.13,0.13,0.13,0.12$ 和 0.12. 规划电网中, 中间中心度高的点为 A19 与 $\mathrm{A} 11$, 其值分别为 0.72 与 0.49 ; 其次为 $\mathrm{B} 12(220 \mathrm{kV})$, $\mathrm{A} 1(220 \mathrm{kV}), \mathrm{A} 9(220 \mathrm{kV}), \mathrm{A} 13(110 \mathrm{kV}), \mathrm{A} 15(220 \mathrm{kV})$, B4(220 kV), a2, B33(220 kV), A12(220 kV), B19(110 $\mathrm{kV})$ 和 $\mathrm{A} 4(110 \mathrm{kV})$, 其值分别为 $0.30,0.29,0.23,0.22$, $0.19,0.18,0.18,0.17,0.13,0.13$ 及 0.12 (图 15 和 16).

规划电网 A19 $(500 \mathrm{kV})$ 等节点有较大中间中心度, 容易出现过载现象. 但是, 规划电网中, 中间中心度 高的 $220 \mathrm{kV}$ 变电站数量大幅增加, 过境荷载在规划
电网节点上的分布更为合理.

(3) 线路过境荷载压力

与节点同理，线路过境荷载压力主要通过“相对 度数中间中心度”进行计算及横向比较. 通过 ucinet 软件中的 Edge(line)Betweenness 计算得知, 现状电网 中 A11-A19 和 A2-A 11 等 2 条线路的中间中心度最高, 分别为 0.19 与 0.15 ; 其次为 A8-A $11, \mathrm{a} 10-\mathrm{a} 32, \mathrm{~A} 1-\mathrm{A} 5$, A2-a1，A7-A11 和 A9-a29 等 6 条线路, 其值分别为 $0.13,0.12,0.12,0.12,0.11,0.11$ 和 0.11(图 17).

规划电网中 A11-b20, A19-b6, A11-A19 和 A11-A15 等 4 条线路中间中心度最高, 分别为 0.14 , $0.12,0.10$ 和 0.10; 其次为 A19-B14, B12-B29, A19B12, A12-B12, A4-b12, A1-b4, A1-b19, A13-B27 和 $\mathrm{B} 23-\mathrm{B} 21$, 分别为 $0.09,0.08,0.08,0.08,0.07,0.07$, 0.07, 0.07 和 0.06(图 18).

对比可见，规划电网中线路的中间中心度有所 下降, 过境荷载压力有所缓解. 同时, 可以看出现状 电网中间中心度高的线路主要集中在 A19(500 kV)及 A11 $(220 \mathrm{kV})$ 变电站, 这在一定程度上也反应出了供 电的层级关系, 规划电网在增加了 $500 \mathrm{kV}$ 变电站 B17 后这种情况并未得到改善, 相反使得 A11 及 A19 周围过载线路更为密集, 因此规划电网在进行与 B17 配套的线路设计时存在缺陷.

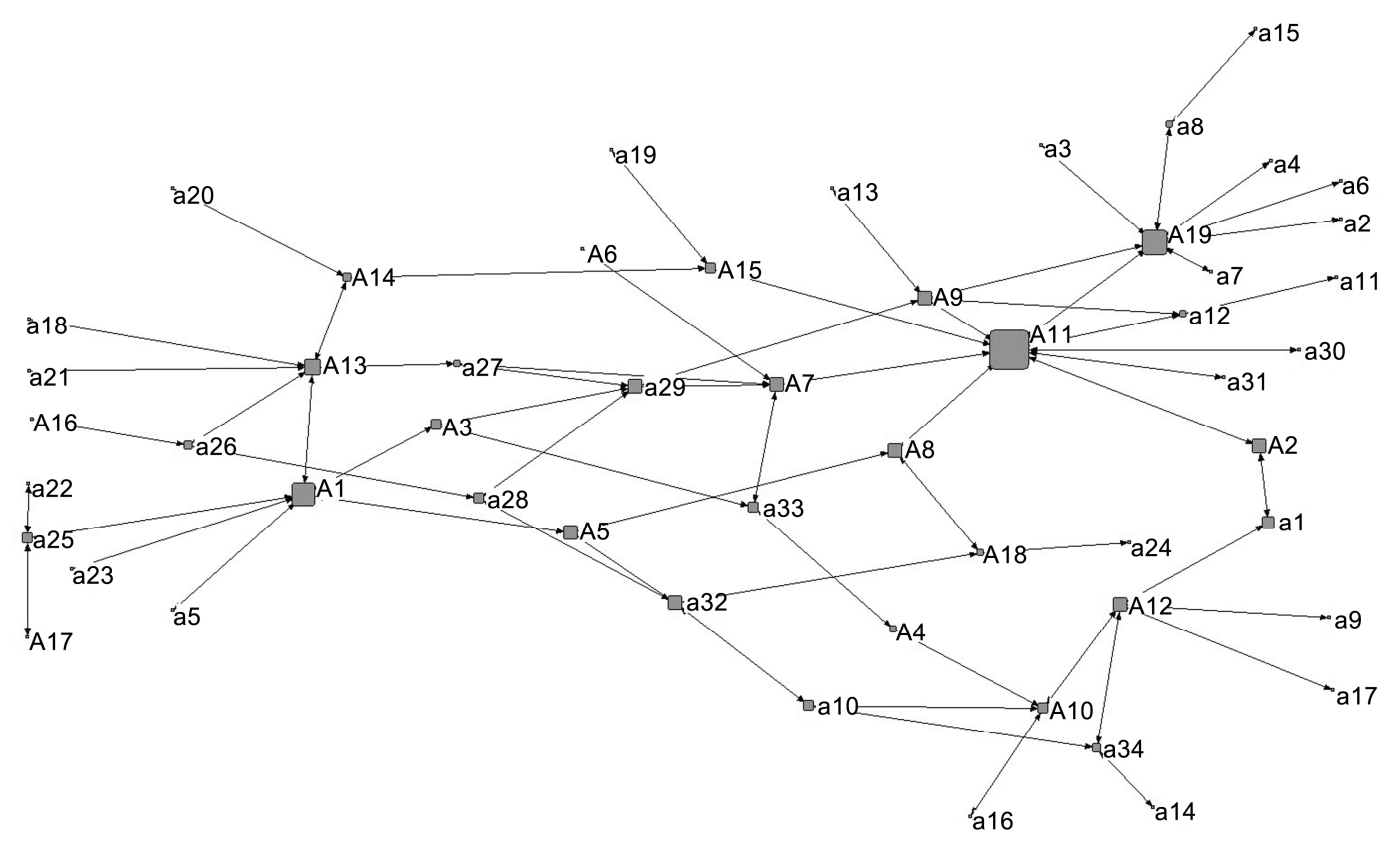

图 15 现状电网点中间中心度 


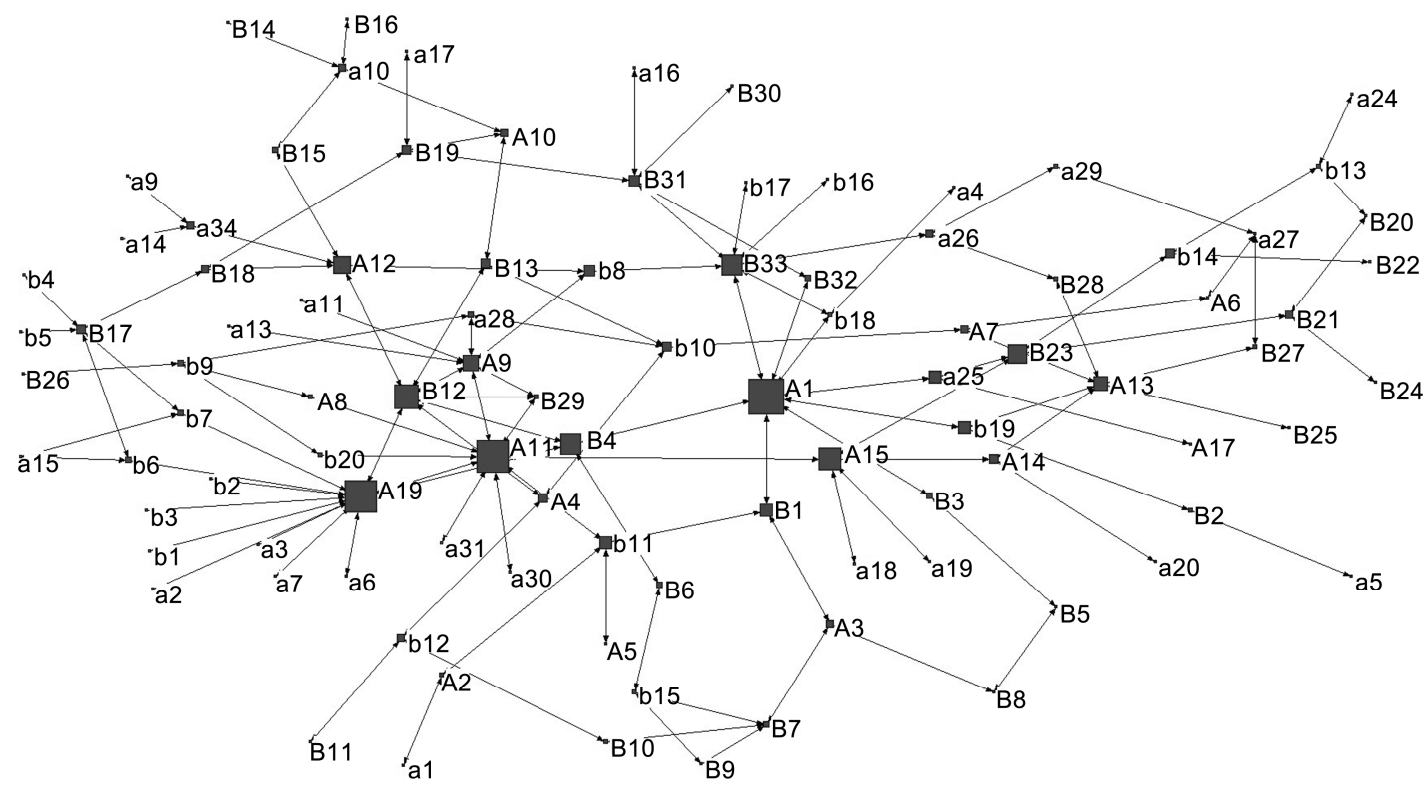

图 16 规划电网点中间中心度

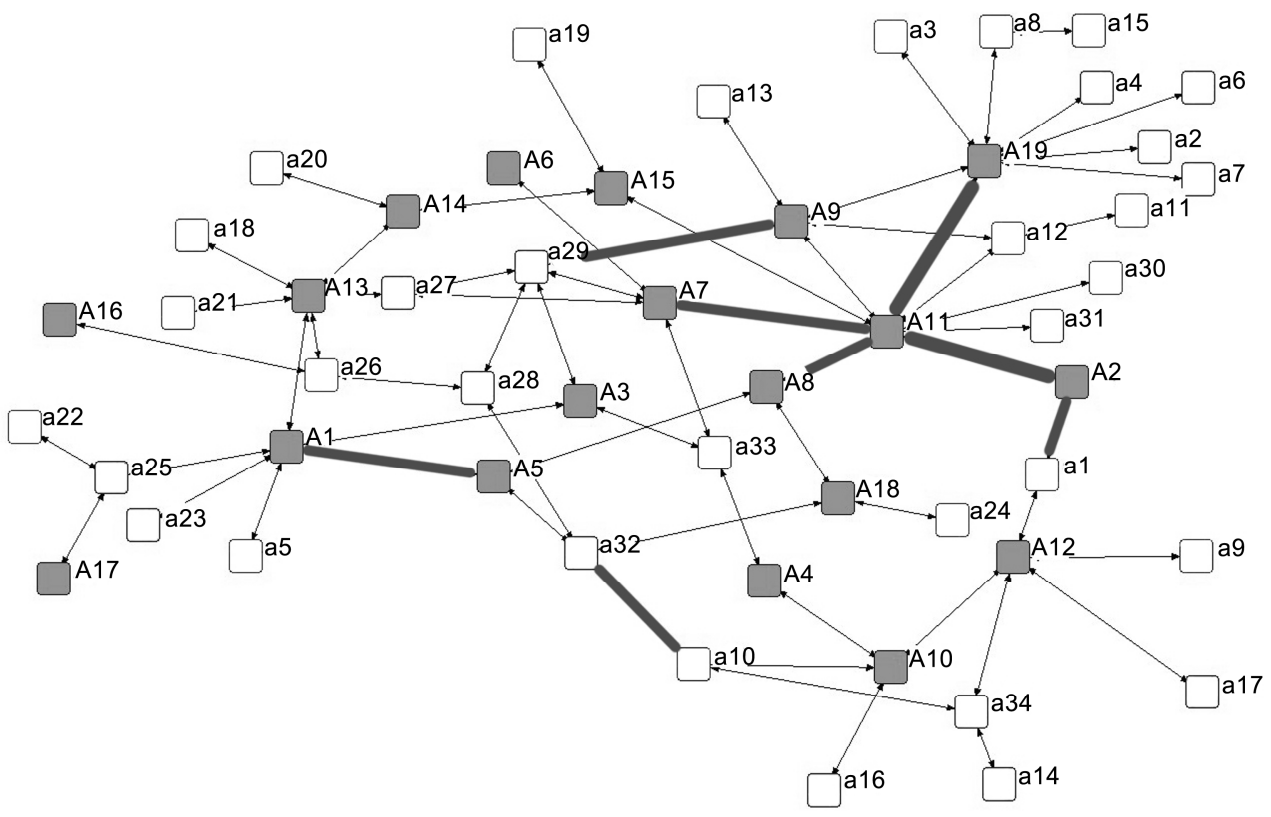

图 17 现状 $220 \mathrm{kV} / 110 \mathrm{kV}$ 电网边的中间中心度

\section{5 结论与讨论}

\section{1 研究结论}

上述计算可知, 万州城区电力基础设施的规划 网络整体上优于现状. 首先, 虽然现状电网在整体结 构上比较完备与紧凑, 但由于线路布置方式等原因, 规划电网具备更高比例的局部稳定结构, 电网的整
体稳定性更强. 其次，荷载在规划网络中的层级分布 更加合理, 尤其在 $220 \mathrm{kV}$ 变电站层面上的荷载分布 更加均衡. 第三，过境荷载在规划电网的全网分布更 加均衡, 过境荷载过载现象在一定程度上有所降低.

但是, 规划电网仍然存在一些问题. 首先, 新增 加的 $500 \mathrm{kV}$ 变电站在网络中的作用并未得到充分体 现，一定程度上存在使用浪费情况. 其次，荷载在 


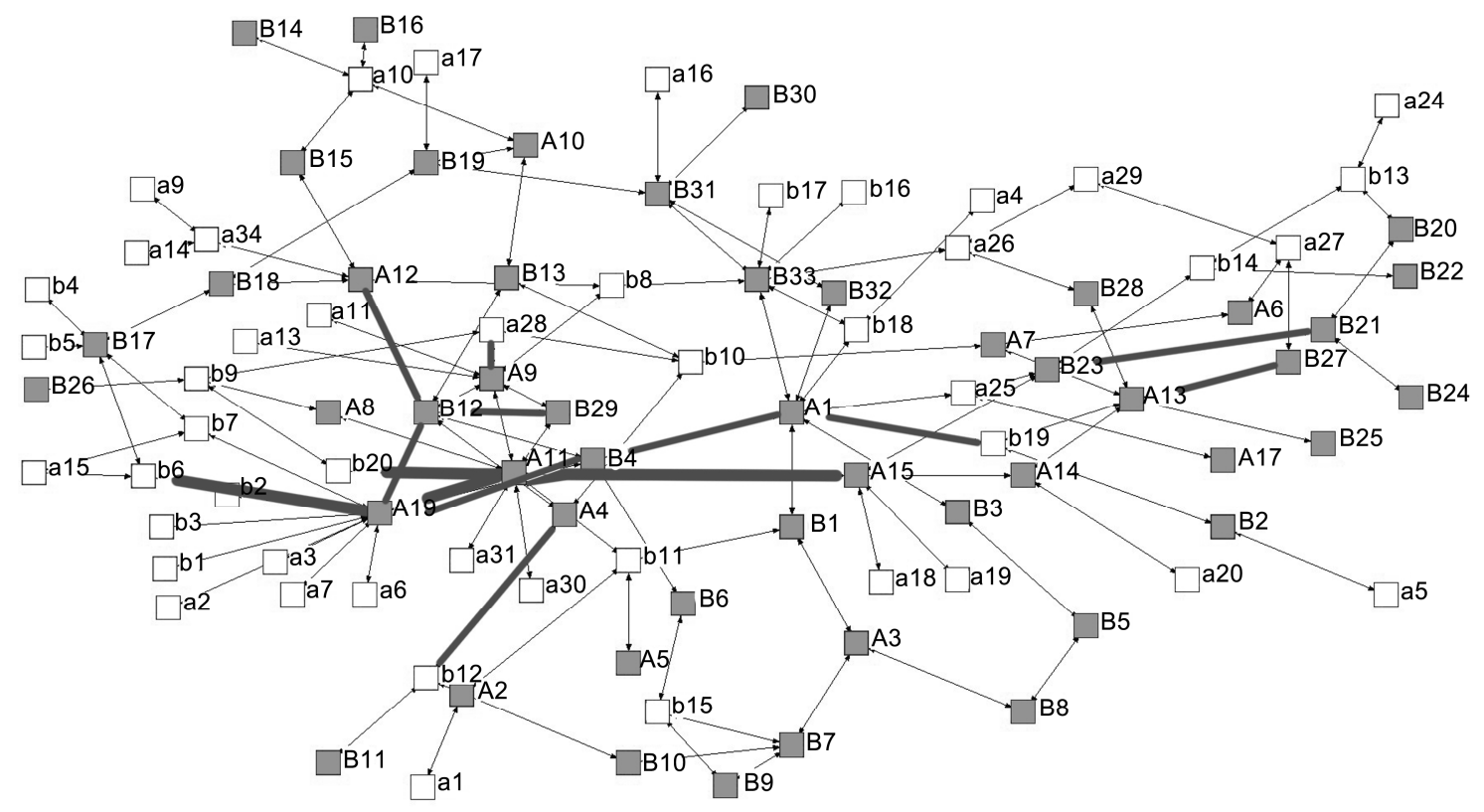

图 18 规划 $220 \mathrm{kV} / 110 \mathrm{kV}$ 电网边的中间中心度

$220 \mathrm{kV}$ 变电站上分布有所改善, 但是对于现状网络 中 $220 \mathrm{kV}$ 变电站的使用情况未产生改善作用, 该部 分变电站仍然存在一定程度上的浪费. 第三, A19(500 kV)等节点存在较大的过境荷载压力. 第四, 新建线路主要围绕着变电站 A19 $(500 \mathrm{kV})$ 及 A11(220 $\mathrm{kV}$ )进行扩展, 并未从整体全局出发考虑问题. 第五, A17 等变电站在网络中处于不稳定部分.

针对以上问题, 有必要从电网拓扑结构设计与 工程加固等两个方面进行着手予以调整和改善.

\section{2 研究展望}

社会网络分析方法能够针对工程型基础设施的 物质构成要素, 挖掘要素个体间的关联关系, 建立整 体的网络拓扑结构, 并就其稳定性和整体性能进行 健康评价. 社会网络分析不仅是一套针对关系或结 构的分析技术, 还是一种理论方法, 有助于我们建立 结构分析观点, 探究隐藏在看似独立的事物表象之 下的网络模式，从而寻找更为本质的客观规律.

\section{参考文献}

1 Dobson I, Chen J, Thorp J. Examining criticality of blackouts in power system models with cascading events. In: Proceedings of the 35th Annual Hawaii International Conference on System Sciences. Hawaii, 2002

2 Surdutovich G, Cortez C V R. Dynamics of "small world" networks and vulnerability of the electric power grid. In: Proceedings of the VIII Symposium of Specialists in Electric Operational and Expansion Planning. Brazil, 2002

3 Wallace W A, MendonOa D, Lee E, et al. Managing disruptions to critical infrastructure interdependencies in the context of the 2001 World Trade Center attack MJ. L. Monday, ed. Beyond September 11th: An account of post-disaster research. Natural Hazards Research and Applications Information Center, Boulder, Colo. 165-198

4 US-Canada Power System Outage Task Force. Causes and Recommendations. Final Report on the August 14, 2003 Blackout in the United States and Canada. 2004

5 OFGEM. Report on Support Investigations Into Recent Blackouts in London and West Midlands. 2004

6 程浩忠, 陈章潮. 电网大面积停电可能性定量分析. 上海交通大学学报, 1999, 12: 1540-1544

7 宋鹏涛, 孟为群, 蒋晓荣, 等. 北京地区“1.19”事故处理及分析. 电网技术, 1996, 8: 61-63

8 唐斯庆, 张弥, 李建设, 等. 海南电网“9.26”大面积停电事故的分析与总结. 电力系统自动化, 2006, 1: 1-7, 16 
9 方锦清, 汪小帆, 郑志刚. 一门崭新的交叉科学: 网络科学(上). 物理学进展, 2007, 3: 239-343

10 Amin M. Toward secure and resilient interdependent infrastructures. J Infrastruct Syst, 2002, 8: 67-75

11 Amarala LA N, Ottino J M. Complex networks: Augmenting the framework for the study of complex systems. Eur Phys J B, 2004, 38: 147-162

12 Carreras B A, Newman D E, Dobson I, et al. Evidence for self-organized criticality in a time series of electric power system blackouts. IEEE Trans Circuits Syst, 2004, 51: 1733-1740

13 Watts D J, Strogatz S H. Collective dynamics of "small-world" networks. Nature, 1998, 393: 440-442

14 Barabasi A L, Albert R. Emergence of scaling in random networks. Science, 1999, 286: 509-512

15 Union for the Coordination of Electricity Transmission (UCTE). Further facts about the system disturbance on 4.11.06. http://www.ucte.org/, 2006-11-06

16 Motter A E, Lai Y C. Cascade-based attacks on complex networks. Phys Rev E, 2002, 66: 065102

17 蔡泽祥, 王星华, 任晓娜. 复杂网络理论及其在电力系统中的应用研究综述. 电网技术, 2012, 11: 114-121

18 柏文洁, 汪秉宏, 周涛. 从复杂网络的观点看大停电事故. 复杂系统与复杂性科学, 2005, 3: 29-37

19 Albert R, Albert I, Nakarado G L. Structural vulnerability of the North American power grid. Phys Rev E, 2004, 69: 025103(R)

20 斯科特. 刘军, 译. 社会网络分析法. 重庆: 重庆大学出版社, 2007

21 邵云飞, 欧阳青燕, 孙雷. 社会网络分析方法及其在创新研究中的运用. 管理学报, 2009, 9: 1188-1193, 1203

22 李二玲, 李小建. 基于社会网络分析方法的产业集群研究一以河南省虞城县南庄村钢卷尺产业集群为例. 人文地理, 2007, 6: 10-15, 128

23 张峰, 杨育, 贾建国, 等. 企业协同生产网络的拓扑特性分析. 重庆大学学报, 2012, 6: 21-27

24 侯望慧, 刘志彪, 岳中刚. 长三角区域经济一体化进程的社会网络分析. 中国软科学, 2009, 12: 90-101

25 陈佳, 匡智锋, 李敏. 一种 Twitter 社区中社会网络分析方法. 计算机工程, 2012, 9: 275-277, 281

26 张炎, 靳继伟, 向罗勇. 相遇时间感知的机会网络社区路由策略. 重庆大学学报, 2013, 6: 137-142

27 毛子骏, 费奇, 欧阳敏, 等. 关联基础设施网络模型研究综述. 计算机科学, 2009, 3: 5-8+33

28 刘军. 社会网络分析导论. 北京: 社会科学文献出版社, 2004

29 吕金虎. 复杂动力网络的数学模型与同步准则. 系统工程理论与实践, 2004, 4: 17-22, 62

30 沈根才. 论电网结构. 中国电力, 1996, 12: 3-8, 71

\title{
Evaluation of urban infrastructure project health based on social network analysis: A case of power grid planning of Wanzhou Districts, Chongqing City
}

\author{
HUANG Yong ${ }^{1,2}$, XIAO Liang ${ }^{2} \&$ HU Yu ${ }^{2}$ \\ ${ }^{1}$ Key Laboratory of New Technology for Construction of Cities in Mountain Area, Chongqing University, Chongqing 400030, China; \\ ${ }^{2}$ Faculty of Architecture and Urban Planning, Chongqing University, Chongqing 400030, China
}

In order to improve the ability of planning, design and health evaluation of infrastructure, such as power, urban traffic and water supply and drainage, and to reduce the urban global risk caused by its failure. Give an example of Chongqing, Wanzhou electric power infrastructure, use social network analysis (SNA) theory and method, build power infrastructure status and network planning respectively, propose the network health evaluation system composed of network complete index and so on, use the network calculation models such as density to assess the health of network topology and its operating state. The empirical study shows, social network analysis method (SNA) can evaluate the network topology and the operation stability of the network objectively.

urban and rural planning, social network analysis (SNA), urban infrastructure, topological structure, health assessment doi: 10.1360/N092014-00071 